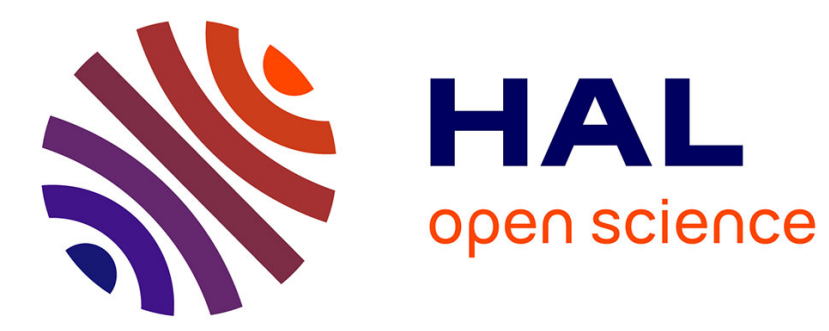

\title{
Fast Linear Attitude Estimation and Angular Rate Generation
}

\author{
Zeliang Zhang, Zebo Zhou, Jin Wu, Shuang Du, Hassen Fourati
}

\section{To cite this version:}

Zeliang Zhang, Zebo Zhou, Jin Wu, Shuang Du, Hassen Fourati. Fast Linear Attitude Estimation and Angular Rate Generation. IPIN 2018 - International Conference on Indoor Positioning and Indoor Navigation, Sep 2018, Nantes, France. pp.1-7, 10.1109/IPIN.2018.8533736 . hal-01928684

\section{HAL Id: hal-01928684 https://hal.inria.fr/hal-01928684}

Submitted on 20 Nov 2018

HAL is a multi-disciplinary open access archive for the deposit and dissemination of scientific research documents, whether they are published or not. The documents may come from teaching and research institutions in France or abroad, or from public or private research centers.
L'archive ouverte pluridisciplinaire HAL, est destinée au dépôt et à la diffusion de documents scientifiques de niveau recherche, publiés ou non, émanant des établissements d'enseignement et de recherche français ou étrangers, des laboratoires publics ou privés. 


\title{
Fast Linear Attitude Estimation and Angular Rate Generation
}

\author{
Zeliang Zhang, Zebo Zhou*, Jin Wu, Shuang Du and Hassen Fourati
}

\begin{abstract}
This paper focuses on the design of an approach to estimate attitude Euler angles and virtual gyroscope output based on a linear Kalman filter and attitude information compensation. First, the rotation transformation is built between the body frame and the reference frame by means of an accelerometermagnetometer triad. The attitude quaternion is invoked for parameterization of orientation which leads to the Kalman filter fusing also the angular rate measurements. Furthermore, to compute the virtual-gyro output in the case of gyroscope failures and obtain bias-free angular rate estimates, virtual gyro Kalman filter is that forms a novel angular rate estimation approach. Some experiment scenarios are given to illustrate the validity and efficiency of the proposed attitude and angular rate estimation approaches.
\end{abstract}

Index Terms-Attitude estimation, Kalman filter, Wahba's problem, angular rate estimation.

\section{INTRODUCTION}

As attitude estimation is crucial for the dead reckoning navigation, great endeavors have been devoted to improving the attitude estimation accuracy and reliability. There are mainly two categories of attitude estimation approaches. One generates attitude estimates along with other navigation quantities, e.g. position, velocity and timing information [1], [2], [3]. The other is solving attitude solution by observation vector pairs matching, typically magnetometers, accelerometers and rate-gyroscopes (MARG) sensors [4], [5], [6]. In aerospace engineering and related geodetic applications, the latter is much more preferred avoiding the strong state parameters coupling existing in the former one [7]. For an attitude measuring system with MARG sensors, the sensor outputs usually contain the system noises due to internal and external factors [4], [5], [8], [9]. Therefore, an efficient fusion method is essential for integrating those complementary sensors. A common approach to multi-sensor data fusion is complementary filters [5], [10], [11]. The frequency domain based filter structure allows for a straightforward implementation without requiring high performance signal processing units [12]. Alternatively, Kalman filtering methods (KF) are intensively used for attitude

Z. Zhang is with the School of Aeronautics and Astronautics, University of Electronic Science and Technology of China (UESTC), Chengdu 611731, China (E-mail: zeliangzzl@163.com).

Corresponding author: Z. Zhou is with the School of Aeronautics and Astronautics, UESTC, Chengdu 611731, China, and with Laboratory of BeiDou Integrated Applications (E-mail: klinsmann.zhou@gmail.com).

J. Wu is with the School of Automation, UESTC, Chengdu 611731, China (E-mail: jin_wu_uestc@hotmail.com).

S. Du is with the School of Aeronautics and Astronautics, UESTC, Chengdu 611731, China (E-mail: sdu@uestc.edu.cn).

H. Fourati is with Univ. Grenoble Alpes, CNRS, Inria, Grenoble INP, GIPSA-Lab, 38000 Grenoble, France (E-mail: hassen.fourati@gipsalab.grenoble-inp.fr). estimation [29]. It involves a set of mathematical equations that produce an optimal recursive solution in the sense of minimum mean square estimation [13]. For nonlinear dynamic systems, the extended Kalman filter (EKF) and unscented Kalman filter (UKF) were developed for achieving the suboptimal solution of nonlinear system models [14], [15]. For those non-Gaussian noise contained systems, critical issues of KF theory are addressed and discussed in [16].

Gyroscope measures the angular rate of a moving object in terms of inertial principles. There is an ordinary differential equation connecting attitude quaternion and angular rate:

$$
\begin{aligned}
\frac{d \boldsymbol{q}_{\omega, k}}{d t} & =\frac{1}{2}[\Omega \times] \boldsymbol{q}_{\omega, k-1} \\
& =\frac{1}{2}\left(\begin{array}{cccc}
0 & -\omega_{x} & -\omega_{y} & -\omega_{z} \\
\omega_{x} & 0 & \omega_{z} & -\omega_{y} \\
\omega_{y} & -\omega_{z} & 0 & \omega_{x} \\
\omega_{z} & \omega_{y} & -\omega_{x} & 0
\end{array}\right) \boldsymbol{q}_{\omega, t-1}
\end{aligned}
$$

where $q_{\omega, k}$ is the quaternion state at the time epoch $k$ and $\omega$ denotes an angular rate component. Combination of accelerometers and magnetometers is popular in compensating for gyroscope drift [8]. Alternatively, fusing accelerometers and magnetometers with gyroscope readings can greatly exempt the noise influences from attitude solution [5]. For the aspect of computation efficiency, many representative methods have been developed, like Gradient Descent Algorithm (GDA) [11], Gauss Newton Algorithm (GNA) [17], [18], LevenbergMarquadt Algorithm (LMA) [31], [32], etc. addressed as the classical Wahbas problem [19]. In addition, compensation strategies are also introduced in the fusion process in the $\mathrm{p}$ resence of magnetic distortion [20] and external accelerometer [4].

Beside attitude determination, angular rate also plays an important role ensuring the flight safety of aero-vehicles, particularly the stabilization system and flight control systems [21]. However, gyroscope may do not work properly under some circumstances, where the output data will be totally erroneous or unavailable. This leads to the divergence for a traditional complementary filter. The observer cannot work properly either. To continuous produce angular rates, virtual gyro is feasible by using accelerometers and magnetometers [22]. However, conventional methods making use of (2) to approximately compute the derivative quantity will inevitably cause large error,

$$
\hat{\dot{\boldsymbol{q}}}=\frac{d \hat{\boldsymbol{q}}}{d t} \approx \frac{\hat{\boldsymbol{q}}_{k}-\hat{\boldsymbol{q}}_{k-1}}{\delta t}
$$




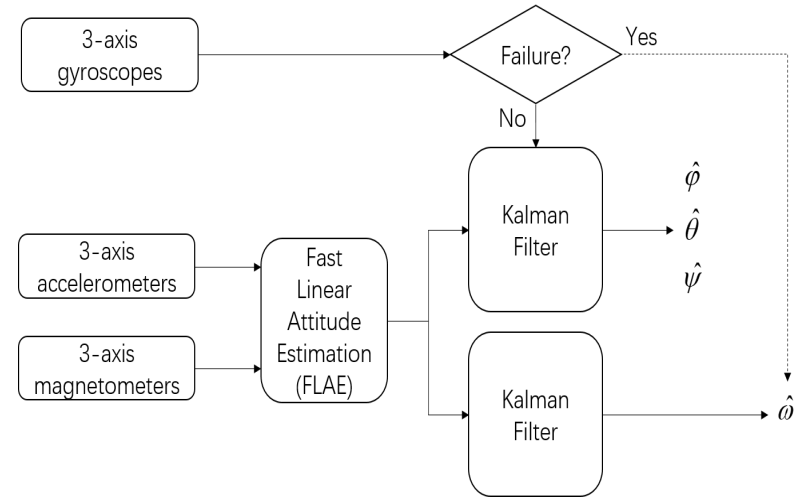

Fig. 1: Attitude calculation and angular rate estimation

This is due to the quaternion estimate derived from accelerometers and magnetometers contains noises which greatly degrade angular rate estimation accuracy.

The paper aims to design an efficient KF structure to accommodate MARG sensors for reliable and accurate attitude as well as angular rate estimation in the scenario of gyroscope failure. Subsequently, the solved attitude quaternion is utilized as the observation model which will be further implemented in KF with the angular rate state model established by gyroscope readings. Conversely, when the gyroscope failure occurs, the attitude information derived from accelerometers and magnetometers will be applied to recover the angular rate information through a dedicated KF. The rest of paper is organized as follows: Section II depicts the principle of attitude determination with MARG sensors. Angular rate estimation strategy is proposed in the case of gyroscope failure in Section III. Section IV presents the experiments and analysis. Finally, conclusions Section V.

\section{Attitude Estimation with MARG Sensors}

In this section, the structure of attitude is constructed based on KF with the Wahba's solution through the combination of tri-axial accelerometers and magnetometers and angular rate from Fig.1.

Estimating relations between body frame (denoted as bframe, right-front-up) and reference frame (denoted as r-frame, east-north-up, i.e. ENU) using accelerometers and magnetometers yields attitude information, which is self-contained in transition matrix. Two sets of vector observation pairs related to the transformation between $\mathrm{b}$ and $\mathrm{r}$ frames are established as follows:

$$
\left\{\begin{array}{l}
\boldsymbol{a}^{\mathrm{b}}=\mathbf{C}_{\mathrm{r}}^{\mathrm{b}} \boldsymbol{a}^{\mathrm{r}} \\
\boldsymbol{a}^{\mathrm{b}}=\mathbf{C}_{\mathrm{r}}^{\mathrm{b}} \boldsymbol{a}^{\mathrm{r}}
\end{array}\right.
$$

where $\boldsymbol{a}^{\mathrm{b}}=\left(a_{x}, a_{y}, a_{z}\right)^{\mathrm{T}}, \mathbf{m}^{\mathrm{b}}=\left(m_{x}, m_{y}, m_{z}\right)^{\mathrm{T}}, \mathbf{a}^{\mathrm{r}}=$ $(0,0,-g)^{\mathrm{T}}, \mathbf{m}^{\mathrm{r}}=\left(0, m_{N}, m_{U}\right)^{\mathrm{T}}$ denote the normalized observation vectors of accelerometers and magnetometers in $\mathrm{b}$ and $\mathrm{r}$ frames, respectively. $\mathbf{C}_{r}^{b}$ is the direction cosine matrix (DCM) (simplified as $\mathbf{C}$ ). The superscript $\mathrm{T}$ denotes the transpose operation.
To find the optimal attitude matrix from multiple vector observations, great deals of efforts are made and most of them can be traced back to the Wahba's problem [19], which aims to minimize the loss function defined by

$$
L(\mathbf{C})=\frac{1}{2} \sum_{i=1}^{n} a_{i}\left\|\mathbf{b}_{i}-\mathbf{C r}_{i}\right\|^{2}
$$

where $\mathbf{C}$ denotes the optimal DCM and $\|\cdot\|$ stands for the Euclidean norm. $\mathbf{b}_{i}$ and $\mathbf{r}_{i}$ denote the normalized observation vectors in body frame $\mathrm{b}$ and reference frame $\mathrm{r}$, respectively. $a_{i}$ is the weight of the $i-$ th vector observation pair satisfying

$$
\sum_{i=1}^{n} a_{i}=1
$$

To solve Wahba's problem and improve the computation efficiency, various of methods have been developed such as Davenport's q-method [23], Markley's fast optimal attitude matrix (FOAM) method [24], Shuster and Oh's quaternion estimator (QUEST) algorithm [25] etc. In this contribution, we introduce the fast linear attitude estimation method (FLAE) to estimate the attitude by using accelerometer/magnetometer vector pairs [27]. It is faster than existing representative algorithms. The conventional Wahba's problem is then converted to the following equations with respect to quaternions as

$$
\left\{\begin{array}{l}
\sqrt{a_{1}} \mathbf{D}_{1}^{\mathrm{b}}=\sqrt{a_{1}} \mathbf{C} \mathbf{D}_{1}^{\mathrm{r}} \\
\sqrt{a_{2}} \mathbf{D}_{2}^{\mathrm{b}}=\sqrt{a_{2}} \mathbf{C D}_{2}^{\mathrm{r}}
\end{array}\right.
$$

in which $\mathbf{D}_{i}=\left(D_{i x}, D_{i y}, D_{i z}\right)(i=1,2)$ are the observation vectors. Obviously, (3) is equivalent to (6). Converting Wahba's problem to an eigenvalue problem as [27]

$$
\mathbf{W} \mathbf{q}=(1+\varepsilon) \mathbf{q}
$$

where $\mathbf{q}=(q 0, q 1, q 2, q 3)^{\mathrm{T}}$ denotes attitude quaternion vector and $\varepsilon$ denotes the error factor. $\mathbf{W}$ is a matrix related to observer vectors and its relevant definition and derivations can be found in [27], [28]. Apparently, as $1+\varepsilon$ is an eigenvalue of $\mathbf{W}$, the problem is shifted to finding the eigenvalue that is closest to 1 . To calculate the eigenvalue of the matrix $\mathbf{W}$, the characteristic polynomial of $\mathbf{W}$ is defined as

$$
f(\lambda) \triangleq \operatorname{det}\left(\mathbf{W}-\lambda \mathbf{I}_{4 \times 4}\right)
$$

$\operatorname{det}(\cdot)$ represents the determinant. Thus it can be further derived to

$$
f(\lambda)=\lambda^{4}+\tau_{1} \lambda^{2}+\tau_{2} \lambda+\tau_{3}
$$


and its symbolic solution is calculated by

$$
\begin{aligned}
& \lambda_{1}=\frac{1}{2 \sqrt{6}}\left(T_{2}-\sqrt{\left.-T_{2}^{2}-12 \tau_{1}-\frac{12 \sqrt{6} \tau_{2}}{T_{2}}\right)}\right. \\
& \lambda_{2}=\frac{1}{2 \sqrt{6}}\left(T_{2}+\sqrt{\left.-T_{2}^{2}-12 \tau_{1}-\frac{12 \sqrt{6} \tau_{2}}{T_{2}}\right)}\right. \\
& \lambda_{3}=-\frac{1}{2 \sqrt{6}}\left(T_{2}+\sqrt{-T_{2}^{2}-12 \tau_{1}+\frac{12 \sqrt{6} \tau_{2}}{T_{2}}}\right) \\
& \lambda_{4}=-\frac{1}{2 \sqrt{6}}\left(T_{2}-\sqrt{-T_{2}^{2}-12 \tau_{1}+\frac{12 \sqrt{6} \tau_{2}}{T_{2}}}\right)
\end{aligned}
$$

where the parameter $\tau_{1}, \tau_{2}, \tau_{3}$ and $T_{2}$ are all detailed in [27]. $\lambda$ is chosen nearest to 1 . In this way, the solving process of is significantly shortened.

Defining $\mathrm{N}$ and transforming $\mathrm{N}$ to $\mathrm{N}^{\prime}$ via elementary row operations, we obtain

$$
\mathbf{N}=\mathbf{W}-\lambda \mathbf{I}_{4 \times 4} \rightarrow \mathbf{N}^{\prime}=\left(\begin{array}{cccc}
1 & 0 & 0 & \chi \\
0 & 1 & 0 & \rho \\
0 & 0 & 1 & v \\
0 & 0 & 0 & \zeta
\end{array}\right)
$$

Thereby, the fundamental solution system is given by:

$$
\tilde{\mathbf{q}}=\left(\begin{array}{c}
q_{0} \\
q_{1} \\
q_{2} \\
q_{3}
\end{array}\right)=\left(\begin{array}{c}
\chi \\
\rho \\
v \\
-1
\end{array}\right)
$$

with its norm of

$$
\|\tilde{\mathbf{q}}\|=\sqrt{\chi^{2}+\rho^{2}+v^{2}+1} .
$$

With the attitude quaternion solution calculated from (12), the linear observation model at epoch $\mathrm{k}$ can be established as:

$$
\mathbf{l}_{k}=\mathbf{H}_{k} \mathbf{x}_{k}+\varepsilon_{k}
$$

where $\mathbf{l}_{k}$ is the observation vector obtained from the (14); $\mathbf{x}_{k}$ denotes the quaternion state to be estimated; the design matrix $\mathbf{H}_{k}$ connecting the state vector to the observation vector becomes unit identity matrix I with dimensions of $4 \times 4 ; \varepsilon_{k}$ is Gaussian distributed with the variance of $\mathbf{Q}_{\varepsilon_{k}}$.

A tri-axial gyroscope senses the 3D angular rate of a certain object with outputs in its body frame as $\omega=\left(\omega_{x}, \omega_{y}, \omega_{z}\right)^{T}$. For a rigid body, its rotation can be alternatively represented by quaternion due to its numerical computation stability. The quaternion and the angular rate are associated with (1). Discretized equation can be modelled as

$$
\mathbf{q}_{\omega, k}=\frac{1}{2} \delta t[\Omega \times] \mathbf{q}_{\omega, k-1}+\mathbf{q}_{\omega, k-1}
$$

where $\delta t$ denotes the sampling interval of gyroscope. Thus (15) can be treated as a dynamic model of the KF,

$$
\mathbf{x}_{k}=\boldsymbol{\Phi}_{k, k-1} \mathbf{x}_{k-1}+\mathbf{w}_{k}
$$

where $\boldsymbol{\Phi}_{k, k-1}$ denotes the transition matrix transferring the previous one state information into that of the current one. According to (15), the transition matrix is then expressed by:

$$
\boldsymbol{\Phi}_{k, k-1}=\frac{1}{2} \delta t[\boldsymbol{\Phi} \times]+\mathbf{I}_{4 \times 4}
$$

$\mathbf{w}_{k}$ denotes the zero-mean process noise with variance of $\mathbf{Q}_{\mathbf{w}_{k}}$. Then the predicted state and its covariance matrix are respectively computed by:

$$
\begin{gathered}
\overline{\mathbf{x}}_{k}=\boldsymbol{\Phi}_{k, k-1} \hat{\mathbf{x}}_{k-1} \\
\mathbf{Q}_{\overline{\mathbf{x}}_{k}}=\boldsymbol{\Phi}_{k, k-1} \mathbf{Q}_{\hat{\mathbf{x}}_{k-1}}\left(\boldsymbol{\Phi}_{k, k-1}\right)^{T}+\mathbf{Q}_{w_{k}}
\end{gathered}
$$

The time update set of KF equations are:

$$
\begin{gathered}
\hat{\mathbf{x}}_{k}=\overline{\mathbf{x}}_{k}+\mathbf{K}_{k}\left(\mathbf{l}_{k}-\mathbf{H}_{k} \overline{\mathbf{x}}_{k}\right) \\
\mathbf{Q}_{\hat{\mathbf{x}}_{k}}=\left(\mathbf{l}_{k}-\mathbf{H}_{k} \overline{\mathbf{x}}_{k}\right) \mathbf{Q}_{\overline{\mathbf{x}}_{k}} \\
\mathbf{K}_{k}=\mathbf{Q}_{\overline{\mathbf{x}}_{k}} \mathbf{H}_{k}^{T}\left(\mathbf{H}_{k} \mathbf{Q}_{\overline{\mathbf{x}}_{k}} \mathbf{H}_{k}^{T}\right)^{-1}+\mathbf{Q}_{\varepsilon_{k}}
\end{gathered}
$$

where $\hat{\mathbf{x}}_{k}=\hat{\mathbf{q}}_{k}$ and $\mathbf{Q}_{\hat{\mathbf{x}}_{k}}=\mathbf{Q}_{\hat{\mathbf{q}}_{k}}$ are KF estimate and its covariance, respectively. $\mathbf{K}_{k}$ is the so-called gain matrix. As time epoch increasing, the quaternion is estimated by implementing (18)-(22).

\section{Angular RATE Estimation With ViRTUAL GYROSCOPE}

\section{A. Virtual gyroscope based Kalman Filter}

When the gyroscope works improperly, angular rate information may be incorrect. Attitude quaternion can still be acquired with the solution to (3), as if accelerometer and magnetometer runs consistently and normally. To smooth the calculated quaternion, a virtual gyroscope based KF is established. Supposing that consecutive first-order derivative of quaternion keeps invariant, the state equation can be modelled as:

$$
\left(\begin{array}{c}
\dot{\mathbf{q}}_{k} \\
\mathbf{q}_{k}
\end{array}\right)=\left(\begin{array}{cc}
\mathbf{I}_{4 \times 4} & \mathbf{0}_{4 \times 4} \\
\delta t \mathbf{I}_{4 \times 4} & \mathbf{I}_{4 \times 4}
\end{array}\right)\left(\begin{array}{c}
\dot{\mathbf{q}}_{k-1} \\
\mathbf{q}_{k-1}
\end{array}\right)+\tilde{\mathbf{w}}_{k}
$$

where the state matrix is chosen with $\tilde{\mathbf{x}}_{k}=\left(\dot{\mathbf{q}}_{k}^{T} \mathbf{q}_{k}^{T}\right)^{T}$. In order to distinguish it from the previous KF state in Section II, we denote the $\mathbf{x}$ with the superscript $\sim$ as the state vector consisting of quaternion and its derivative. Thereby, the corresponding transition matrix is

$$
\tilde{\boldsymbol{\Phi}}_{k, k-1}=\left(\begin{array}{cc}
\mathbf{I}_{4 \times 4} & \mathbf{0}_{4 \times 4} \\
\delta t \mathbf{I}_{4 \times 4} & \mathbf{I}_{4 \times 4}
\end{array}\right)
$$

and $\tilde{\mathbf{w}}_{k}$ denotes the zero-mean process noise with covariance matrix of $\mathbf{Q}_{\tilde{\mathbf{w}}_{k}}$.

The quaternion vector obtained from the (12) is still used as observation vector, thus linear observation model at epoch $k$ is established as:

$$
\mathbf{l}_{k}=\tilde{\mathbf{H}}_{k} \tilde{\mathbf{x}}_{k}+\tilde{\varepsilon}_{k}
$$

where the design matrix $\tilde{\mathbf{H}}_{k}$ becomes such as:

$$
\tilde{\mathbf{H}}_{k}=\left(\begin{array}{ll}
\mathbf{0}_{4 \times 4} & \mathbf{I}_{4 \times 4}
\end{array}\right)
$$

and $\tilde{\varepsilon}_{k}$ denotes the zero-mean observation noise with variance of $\mathbf{Q}_{\tilde{\varepsilon}_{k}}$. The new Kalman filter's implementation process is similar to $(18)-(22)$. 


\section{B. Angular rate estimation}

With quaternion and its first derivative obtained from new $\mathrm{KF}$, the angular rate can be estimated according to the arithmetic of quaternion as follows:

$$
\hat{\Omega}_{k}=2 \hat{\dot{\mathbf{q}}}_{k} \otimes \hat{\mathbf{q}}_{k-1}^{*}
$$

where $\otimes$ represents the quaternion product, and the superscript * stands for the conjugate of quaternion. It can also be solved by means of Euler Angles according to the relation between quaternion and Euler Angles as follows:

$$
\begin{gathered}
\hat{\phi}=\arctan \frac{2\left(\hat{q}_{2} \hat{q}_{3}+\hat{q}_{0} \hat{q}_{1}\right)}{\hat{q}_{0}^{2}-\hat{q}_{1}^{2}-\hat{q}_{2}^{2}+\hat{q}_{3}^{2}} \\
\hat{\theta}=\arcsin \left(2\left(\hat{q}_{0} \hat{q}_{2}-\hat{q}_{1} \hat{q}_{3}\right)\right) \\
\hat{\psi}=\operatorname{arttan} \frac{2\left(\hat{q}_{1} \hat{q}_{2}+\hat{q}_{0} \hat{q}_{3}\right)}{\hat{q}_{0}^{2}+\hat{q}_{1}^{2}-\hat{q}_{2}^{2}-\hat{q}_{3}^{2}}
\end{gathered}
$$

$\hat{\phi}, \hat{\theta}$ and $\hat{\psi}$ denote roll, pitch and yaw estimated angles, respectively. Then the angular rate is acquired by differential operation of Euler angles referring to its definition that:

$$
\begin{gathered}
\hat{\omega}_{x, k}=\frac{d \hat{\phi}}{d t} \approx \frac{\hat{\phi}_{k}-\hat{\phi}_{k-1}}{\delta t} \\
\hat{\omega}_{y, k}=\frac{d \hat{\theta}}{d t} \approx \frac{\hat{\theta}_{k}-\hat{\theta}_{k-1}}{\delta t} \\
\hat{\omega}_{z, k}=\frac{d \hat{\psi}}{d t} \approx \frac{\hat{\psi}_{k}-\hat{\psi}_{k-1}}{\delta t}
\end{gathered}
$$

\section{EXPERIMENTS AND RESULTS}

An experimental platform is designed to conduct various experiments. Uranus 2 produced by Beijing Beyond Core Electronic Technology Co., Ltd. provides the output of tri-axial gyroscope, accelerometer and magnetometer. TTL to USB module is applied so that the sensor data from Uranus2 can be saved into computer. MATLAB r2016 is used for data processing and analysis. We put the MARG element at rest for a while, then we rotate it, trying to keep around the center of the mass. The samples are collected by using the above equipment and the raw data is drawn in Fig. 2.

Figure 3 shows the solution to attitude quaternion obtained with acceleration and magnetometer, which is very noisy. Time consumption of QUEST and FLAE is presented in Table I. Obviously, Quaternion gets more smooth and has better precision after MARG-KF, when gyroscope measurements are fused. The corresponding Euler angles are presented in Fig. 4. Three methods are employed to estimate angular rate in the case of absent gyroscope readings for comparison purpose:

1) Non-filtered quaternion (NFQ) with (2) and (27);

2) Non-filtered Euler angles (NFEA) with (28)-(33);

3) Our Proposed virtual gyroscope KF (VGKF).

Figure 5 shows the derivative of quaternion in (2) and the result of NFQ is shown in Fig. 6. The estimated quaternion derivatives are too noisy thus cover the true signals. Therefore, it is not suitable for calculating angular rate.

Seen from Fig. 7, NFEA is also unreliable to convert the quaternions directly to Euler angles without filtering. The
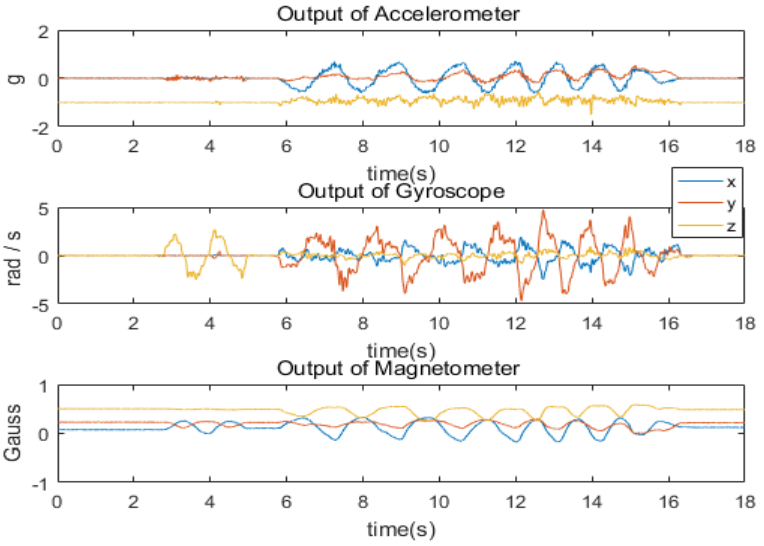

Fig. 2: Raw data from Uranus2.

TABLE I: Time consumption of QUEST and FLAE

\begin{tabular}{|c|c|c|}
\hline \multicolumn{3}{|c|}{ Time Consumpution $\left(\times 10^{-4} s\right)$} \\
\hline & QUEST & $\boldsymbol{F L A} \boldsymbol{E}$ \\
\hline 1 & 0.67 & 0.54 \\
2 & 0.79 & 0.49 \\
3 & 0.92 & 0.47 \\
4 & 0.88 & 0.54 \\
5 & 0.72 & 0.46 \\
6 & 0.73 & 0.55 \\
7 & 0.60 & 0.34 \\
8 & 0.74 & 0.42 \\
9 & 0.80 & 0.39 \\
10 & 0.63 & 0.21 \\
11 & 0.79 & 0.44 \\
12 & 1.43 & 0.77 \\
\hline
\end{tabular}
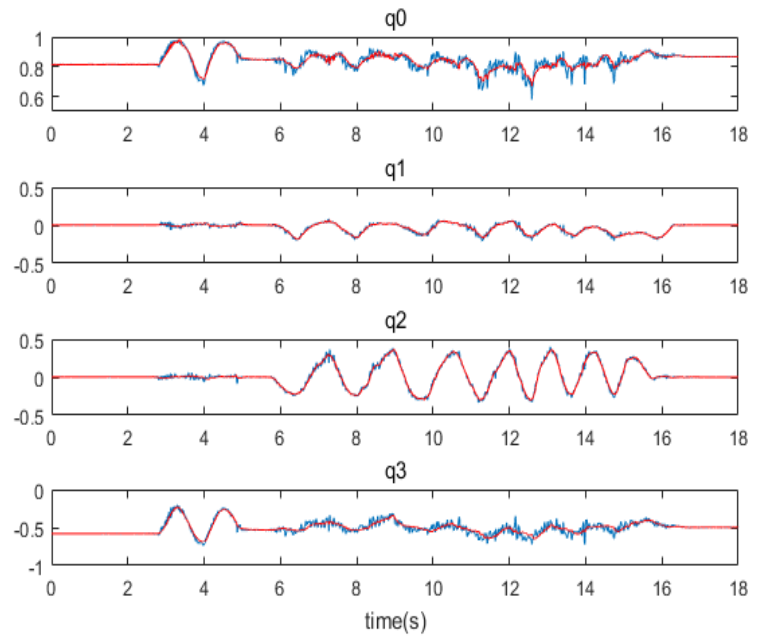

Fig. 3: Quaternion of Wahba's solution calculated with accelerometer and magnatometer (blue line) and MARG-KF (red line) 

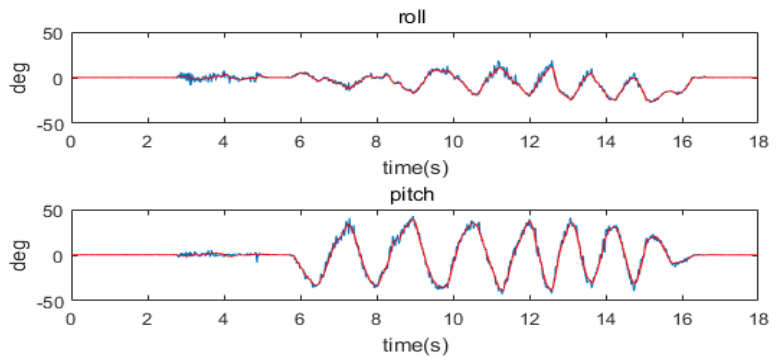

yaw

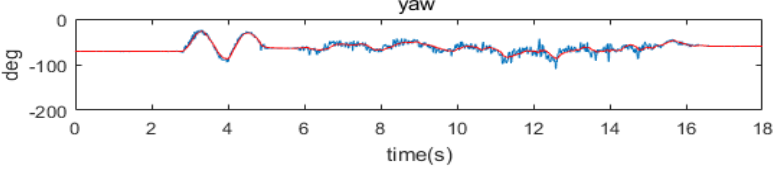

Fig. 4: Euler angles relative to the quaternion Wahba's solution (blue line) and MARG-KF (red line).
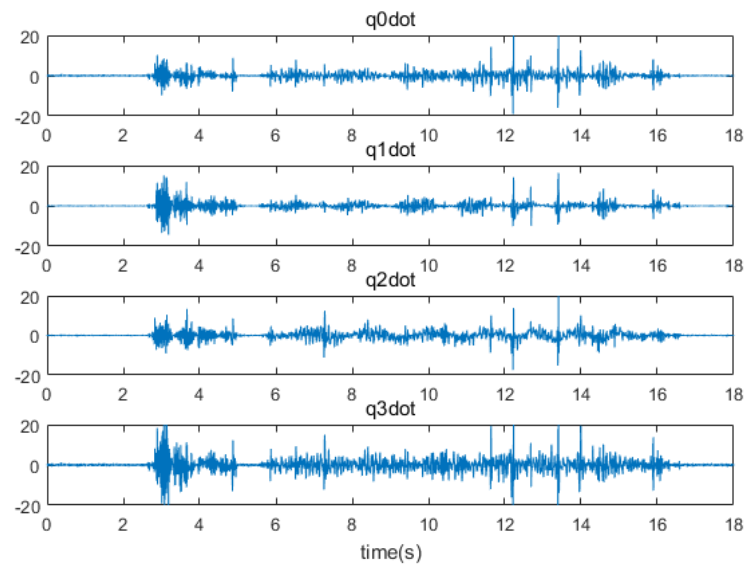

Fig. 5: The derivative of quaternion in (2).
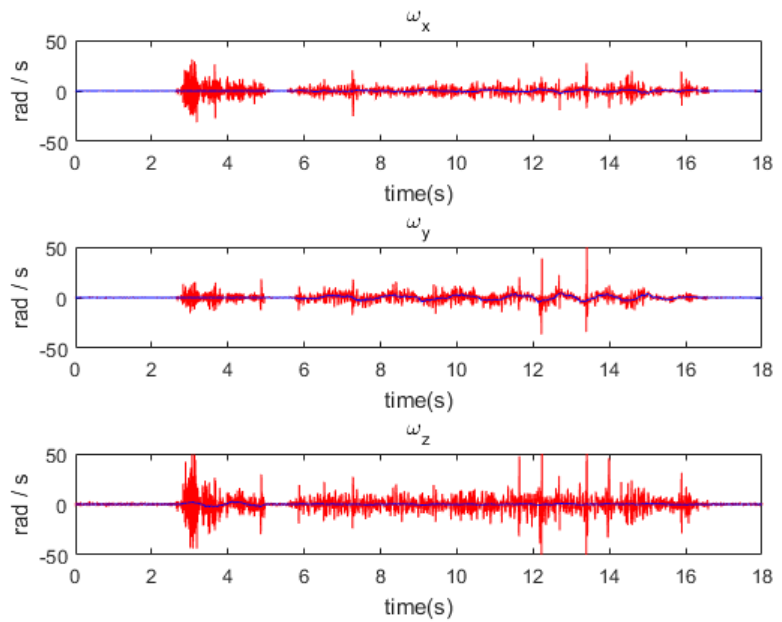

Fig. 6: Angular rates of NFQ (red line) and references (blue line)
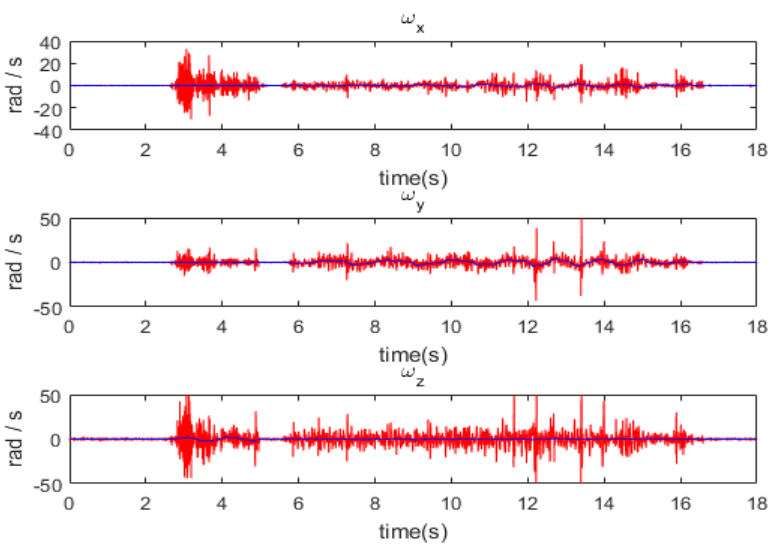

Fig. 7: Angular rates of NFEA (red line) and references (blue line)
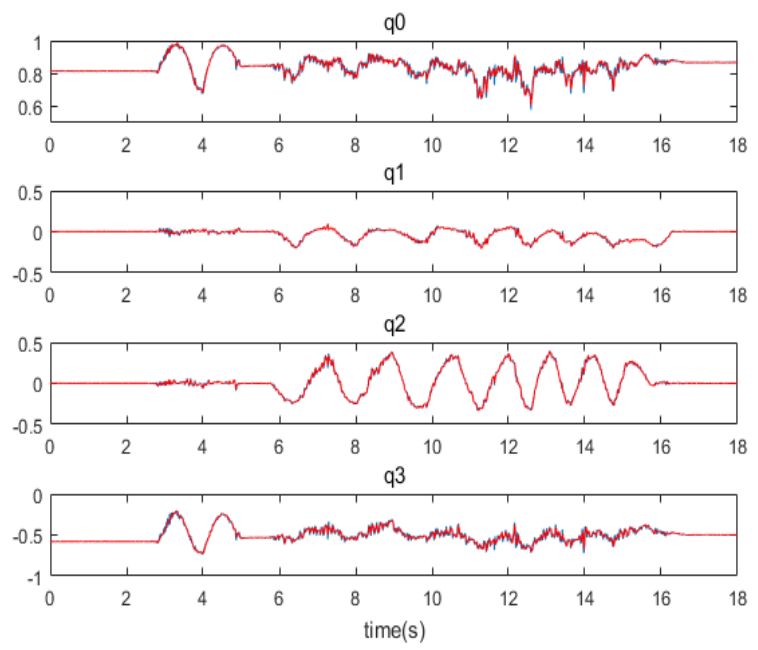

Fig. 8: Quaternion of Wahba's solution (blue line) and VGKF (red line)

results and reasons are very similar to NFQ. The reason why angular rate cannot be directly calculated is that the two consecutive quaternions and their first derivations are very sensitive to the quaternion errors which will further be significantly augmented thus analytically propagate into the estimated results. The proposed VGKF is designed to solve such problem. Figure 8 shows the quaternion solution of the proposed VGKF. Though it is not as good as the original gyroscope readouts, its uncertainty has been extensively minimized. Likewise, as shown in Fig. 9, the accuracy of its derivative has been significantly improved as well. It should also be noted that VGKF is effective and reaches a good consistency with the actual angular rate from gyroscope readings as shown in Fig. 10. 

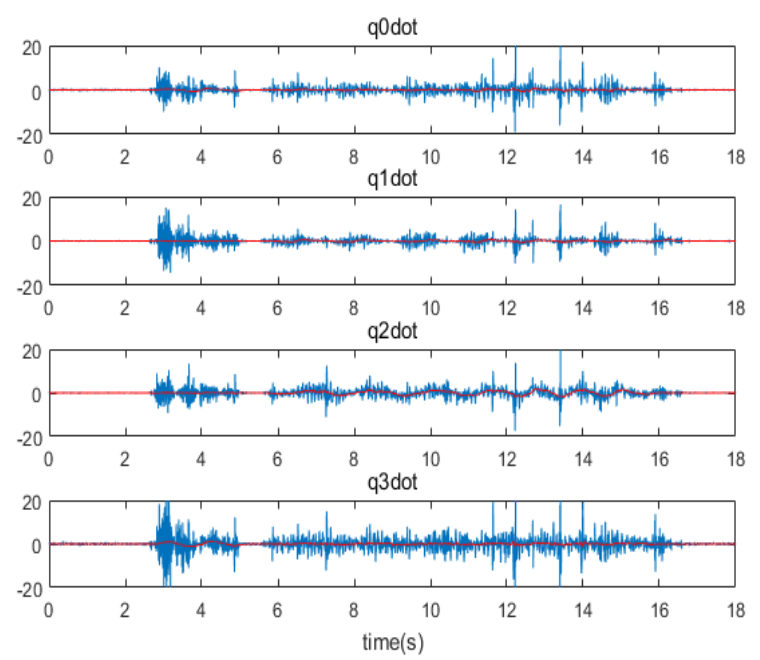

Fig. 9: The derivative of quaternion in (20) (blue line) and that in VGKF (red line)
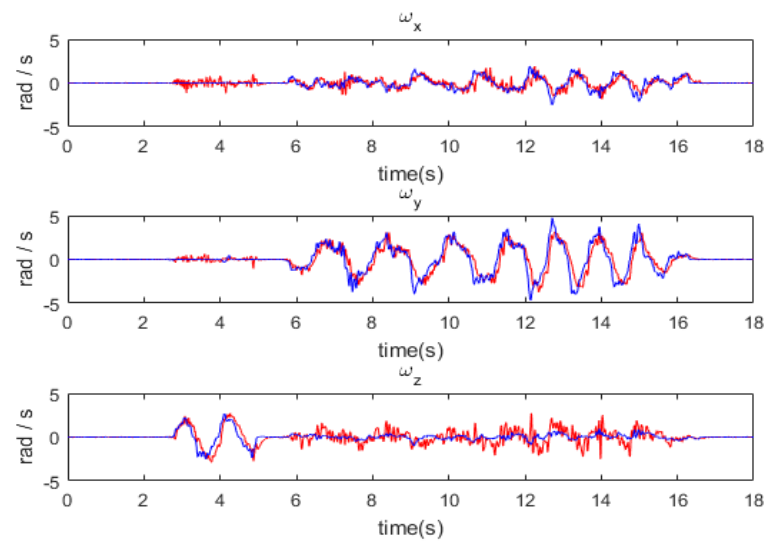

Fig. 10: Angular rates of VGKF (red line) and real gyroscope readings (blue line)

\section{CONCLUSION}

The state model of attitude is constructed based on Kalman Filter theory. Two different structures of Kalman filtering are designed to obtain accurate attitude estimates as well as the angular rates that significantly determines the robustness of the system in case of failures and faults in rate-gyroscope i.e. the solution to the angular rate estimation is also put forward for the absence of gyroscope data. Experimental results show that the proposed solution is effective. In the future, fault detection, isolation and error compensation will be comprehensively investigated and applied to make MARG sensor's fusion results more accurate and reliable.

\section{ACKNOWLEDGMENT}

This work is supported by the National Natural Science Funds of China (No. 41604025 and 41704029), the State Key Laboratory of Geodesy and Earth's Dynamics (Institute of
Geodesy and Geophysics, CAS, SKLGED2018-3-2-E) and the Key Laboratory of Precise Engineering and Industry Surveying of National Administration of Surveying, Mapping and Geoinformation (PF2015-11).

\section{REFERENCES}

[1] Tasdemir E, Kurz L. and Noll T. G. Efficient computation of the position, velocity and time estimation algorithm on a GNSS processor using interpolation, Satellite Navigation Technologies and European Workshop on Gnss Signals and Signal Processing IEEE, pp. 1-7, 2012.

[2] Zhou Z. , Li Y., Zhang J. and Rizos C. Integrated navigation system for a low-cost quadrotor aerial vehicle in the presence of rotors influences. Journal of Surveying Engineering, vol. 143, no. 1, 2017.

[3] Britting, K. R. Inertial navigation system analysis. Avionics. 1971.

[4] Hemerly E.M., Maciel B.C.O., Milhan A.D.P., Schad V.R. Attitude and heading reference system with acceleration compensation. Aircraft Engineering and Aerospace Technology, vol. 84(2), pp. 87-93 2012.

[5] Wang Y., Li N., Chen X. and Liu M. Design and implementation of an AHRS based on MEMS sensors and complementary filtering. Advances in Mechanical Engineering, pp. 1-11, 2014.

[6] Batista P., Silvestre C., Oliveira P. and Cardeira C. Low-cost Attitude and Heading Reference System: Filter design and experimental evaluation. IEEE International Conference on Robotics and Automation, pp. 26242629, 2010.

[7] Zhou Z., Wu J., Wang J. and Fourati H. Optimal Recursive and Sub-Optimal Linear Solutions to Attitude Determination from Vector Observations for GNSS/Accelerometer/Magnetometer Orientation Measurement. Remote sensing, vol. 10(3), 377; 2018.

[8] Suh Y.S. Orientation estimation using a quaternion-based indirect kalman filter with adaptive estimation of external acceleration. IEEE Transactions on Instrumentation and Measurement, vol. 59(12), pp. 3296-3305, 2010.

[9] Thienel J. and Sanner R.M. A coupled nonlinear spacecraft attitude controller/observer with an unknown constant gyro bias. Decision and Control, vol. 4, pp. 3441-3446, 2001.

[10] Wu J., Zhou Z., Chen J., Fourati H. and Li R. Fast Complementary Filter for Attitude Estimation Using Low-Cost MARG Sensors. IEEE Sensors Journal, vol. 16(18), pp. 6997-7007, 2016.

[11] Wang L., Fu L. and Hu X. A nonlinear complementary filter approach for MAV 3D-attitude estimation with low-cost MARG/ADS. Position, Location and Navigation Symposium IEEE, pp. 42-47, 2016.

[12] Merhav S. Aerospace Sensor Systems and Applications, New York:Springer-Verlag, vol. 9(1), 1998.

[13] Zhou Z., Li Y., Liu J. and Li G. Equality constrained robust measurement fusion for adaptive Kalman filter based heterogeneous multi-sensor navigation. IEEE Transactions on Aerospace and Electronic Systems, vol. 49(4), pp. 2146-2157, 2013.

[14] Kandepu R., Foss B. and Imsland L. Applying the unscented Kalman filter for nonlinear state estimation. Journal of Process Control, vol. 18(7C8), pp. 753-768, 2008.

[15] Marins J.L., Yun X., Bachmann E.R. and Mcghee R.B. An extended Kalman filter for quaternion-based orientation estimation using MARG sensors. International Conference on Intelligent Robots and Systems, vol. 4, pp. 2003-2011, 2001.

[16] Zhou Z., Wu J, Li Y., Fu C. and Fourati H. Critical issues on Kalman filter with colored and correlated system. Asian Journal of Control, vol. 20(1), pp. 1-15, 2018.

[17] Tian Y., He W. and Tan J. An adaptive-gain complementary filter for real-time human motion tracking with MARG sensors in free-living environments, IEEE Transactions on Neural Systems and Rehabilitation Engineering, pp. 254-264, 2013.

[18] Liu F., Li J., Wang H. and Liu C. An improved quaternion GaussC Newton algorithm for attitude determination using magnetometer and accelerometer, China J. Aeronautics, vol. 27(4), pp. 986-993, 2014.

[19] Wahba G. A Least Squares Estimate of Satellite Attitude[J]. Siam Review, vol. 7(3), pp. 409-409. 1965.

[20] Roetenberg D., Luinge H.J., Baten C.T.M. and Veltink P.H. Compensation of magnetic disturbances improves inertial and magnetic sensing of human body segment orientation. IEEE Transactions on Neural Systems and Rehabilitation Engineering A Publication of the IEEE Engineering in Medicine and Biology Society, vol. 13(3), pp. 395, 2005. 
[21] Zhao C. Design of an Angular-Rate Compensation Based Strap-down Antenna Stabilization System. Electronics Optics and Control, 2010.

[22] Mike S. Building a virtual gyro. https://blog.nxp.com/sensors/buildinga-virtual-gyro, 2013.

[23] Choukroun D., Bar-Itzhack I.Y. and Oshman Y. Novel quaternion kalman filter. Aerospace and Electronic Systems IEEE Transactions on, vol. 42(1), pp.174-190, 2002.

[24] Markley F. L. Attitude determination from vector observations: A fast optimal matrix algorithm, J. Astron. Sci., vol. 41(2), pp. 261-280, 1993.

[25] Shuster M. D. and Oh S. D. Three-axis attitude determination from vector observations. J Guidance Control Dynam, vol. 4(1), pp. 70-77, 1981.

[26] Vasconcelos J F, Cunha R, Silvestre C and Oliveira P. Stability of a nonlinear attitude observer on $\mathrm{SO}(3)$ with nonideal angular velocity measurements. Control Conference. IEEE, pp. 2769-2774, 2009.

[27] Wu, J., Zhou, Z., Gao, B., Li, R., Cheng, Y. and Fourati, H. Fast Linear Quaternion Attitude Estimator Using Vector Observations. IEEE Transactions on Automation Science and Engineering, vol. 15(1), pp. 307-319, 2018.

[28] Caruso M.J. Applications of magnetic sensors for low cost compass systems. Position Location and Navigation Symposium, pp. 177-184, 2002.

[29] Welch G, Bishop G. An Introduction to the Kalman Filter. University of North Carolina at Chapel Hill, 2001.

[30] Vasconcelos J.F., Cunha R., Silvestre C. A nonlinear position and attitude observer on SE(3) using landmark measurements. Systems and Control Letters, vol. 59(3), pp. 155-166, 2010.

[31] Fourati H., Manamanni L. A. and Handrich Y. A nonlinear ?ltering approach for the attitude and dynamic body acceleration estimation based on inertial and magnetic sensors: Bio-logging application, IEEE Sensors Journal, vol. 11(1), pp. 233-244, 2011.

[32] Fourati H., N. Manamanni L. A. and Handrich Y. Complementary observer for body segments motion capturing by inertial and magnetic sensors, IEEE/ASME Trans. Mechatronics, vol. 19(1), pp. 149-157, 2014.

[33] Park S. and Hong S.K. Angular rate estimation using a distributed set of accelerometers. Sensors, vol. 11(11), pp. 10444-10457, 2011.

[34] Liu C., Yu S., Zhang S., Yuan, X. and Liu S. An effective unscented Kalman filter for state estimation of a gyro-free inertial measurement unit. Position, Location and Navigation Symposium - PLANS 2014, pp.230-235, 2014. 